\title{
Effect of oil film behavior on roller surface on cooling in traction drives
}

\author{
Naoki MURAOKA* and Masayuki OCHIAI* \\ *Department of Mechanical Engineering, Tokai University \\ 4-1-1Kitakaname, Hiratsuka, Kanagawa 259-1292, Japan \\ E-mail: ochiai-m@tsc.u-tokai.ac.jp
}

Received: 12 June 2019; Revised: 23 September 2019; Accepted: 14 November 2019

\begin{abstract}
This paper describes the relationship between the oil film behavior and cooling effect of traction drive roller experimentally. Traction drives are transmission systems where the power is transmitted by shear stress of lubrication oil on the contact point between the rollers. They have advantages regarding noise, vibration, and high-speed operation compared with gears. On the other hand, when the power is transmitted, the heat generation occurs caused by the creep and spin at contact points. This leads to decrease of traction force and then leads to reduction of mechanical efficiency of power transmission. Thus, traction drives need the additional cooling device or the supplying of a large amount of cooling oil. However, these cooling method causes an increase of device weight, device cost, and energy consumption. From this background, the authors focused on the cooling effect of oil film behavior on traction roller. In this study, the oil film behavior was observed, and oil film temperatures were measured. As a result, it is found that oil film temperatures are affected by the oil splashing from the roller surface and the oil film distribution. Further, the authors proposed a new geometry of the roller surface which can expect to increase the cooling effect by the oil film.
\end{abstract}

Keywords : Traction drives, Oil film temperature, Cooling effect, Oil splashing, Oil film distribution

\section{Introduction}

Traction drives are transmission systems that can transmit rotating power using the shear stress of lubrication oil in the contact points between the two rollers. Noise and vibration of these systems are less than those of gears. On the other hand, traction coefficient decreases with an increase in oil film temperature due to heat generation of oil at the contact point (Tanaka, 1989). Therefore, the traction oil is cooled with a cooling device or by a supply of a large amount of oil (Machida, 1990). However, there are some problems using these cooling methods, for example, heavy weight, high cost, and energy consumption. Therefore, the authors focused on the cooling effect of oil film behavior on the traction roller surface. In previous studies, A. Dewinter and H. Blok investigated the cooling effect of the oil splashing in the spur gear through analysis (Dewinter et al., 1974). In addition, the relationship between the amount of oil splashing and the cooling effect was studied, and was estimated the effective supply method for cooling (Yamada et al., 1981). Further, the relationship between the oil film behavior and the cooling effect were investigated in centrifugal lubrication of spur gears (Obata et al., 1994). Moreover, the oil splashing from the gear teeth surface were visualized and the effect of the splashing oil on cooling the gear was discussed. On the other hand, in traction drives, the cooling effects have been studied by using CFD analysis and how the flow velocity of the supply oil affects an oil film temperature was discussed (Suwa et al., 2018). However, there are no studies investigating the relationship between oil film behavior and cooling effect in traction drives. Therefore, in this study, measurement of an oil film temperature, visualization of oil splashing and visualization of oil film distribution on the roller surface were conducted and the mechanism of cooling effect of traction roller was considered. Finally, we propose a new geometry of roller for effective cooling of traction drives. 


\section{Experimental apparatus and method \\ 2.1 Experimental apparatus}

Figure 1 shows the experimental apparatus used in this study, consisting of a sapphire grass disk, a curvature shaped roller, two motors, a feed screws, and two spindles. The roller is pressed by the disk by using the feed screws during the experiment. In addition, the apparatus can replicate the heat generation of oil at the contact point by using the peripheral velocity different rollers and the disk. Figure. 2 shows the definition of the rolling direction in this paper. In the case that the oil engulfs between the roller and the disk, it is called "downward." In contrast, the opposite direction of downward is called "upward." In this experiment, results of oil film temperature and visualization results of oil splashing were compared in the case of the upward with the downward.

\subsection{Measurement of oil film temperature}

Table1 shows the experimental conditions of oil film temperature measurement, and Fig. 3 shows the method of the thermocouple setting. The oil film temperature distributions on the roller surface are measured with 12 thermocouples as shown in Fig. 3(a) and the position is $10 \mu \mathrm{m}$ away from the roller surface as shown in Fig. 3(b).

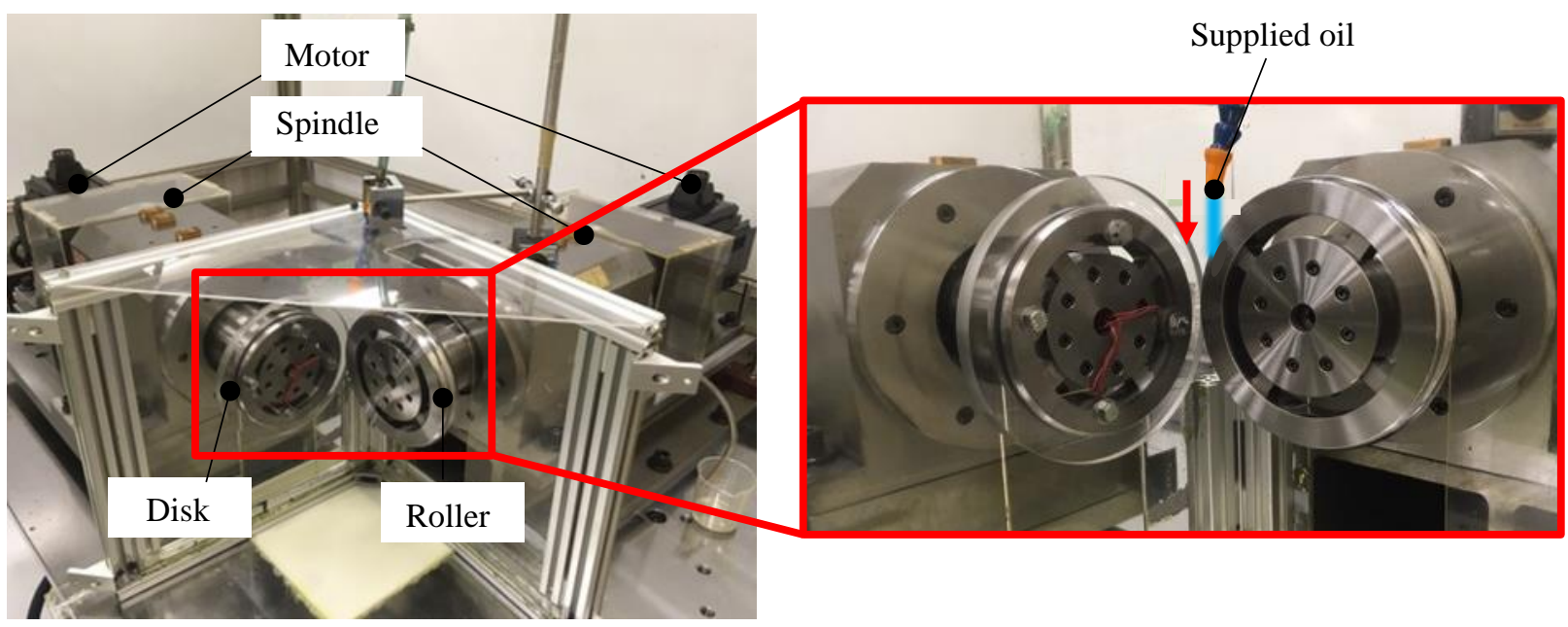

Fig. 1 Experimental apparatus is consisted of the disk, the roller, two spindles, and two motors.

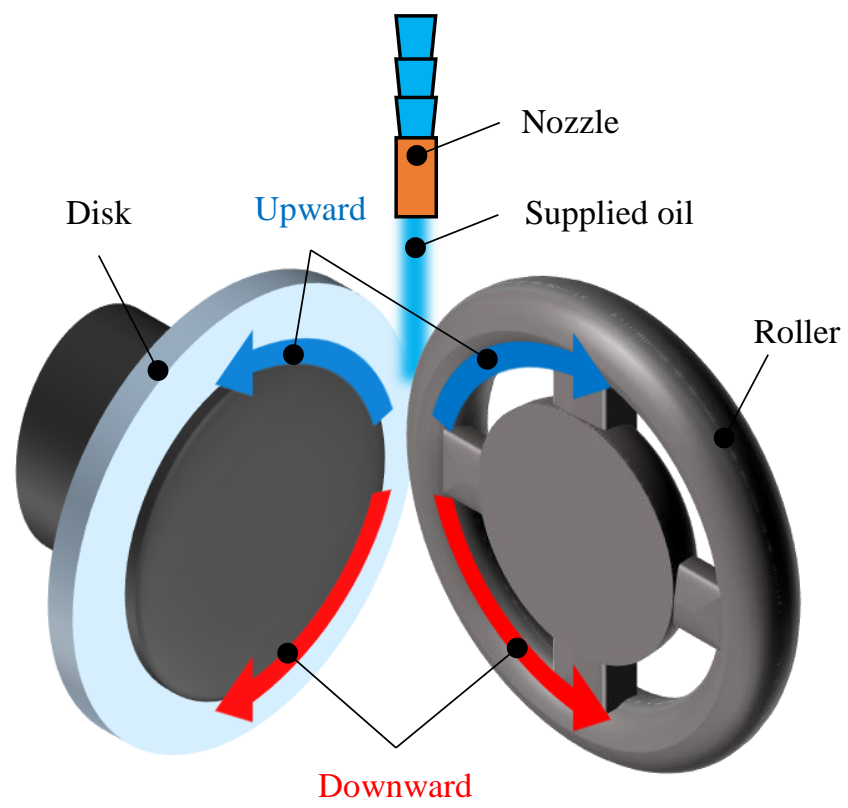

Fig. 2 The downward is rolling direction that oil is engulfed by a roller and a disk. 


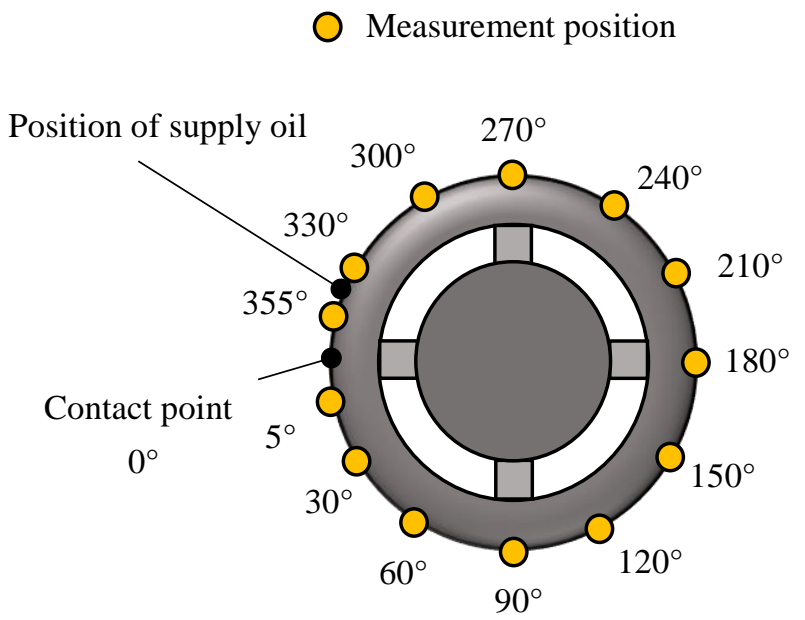

(a) Measurement point

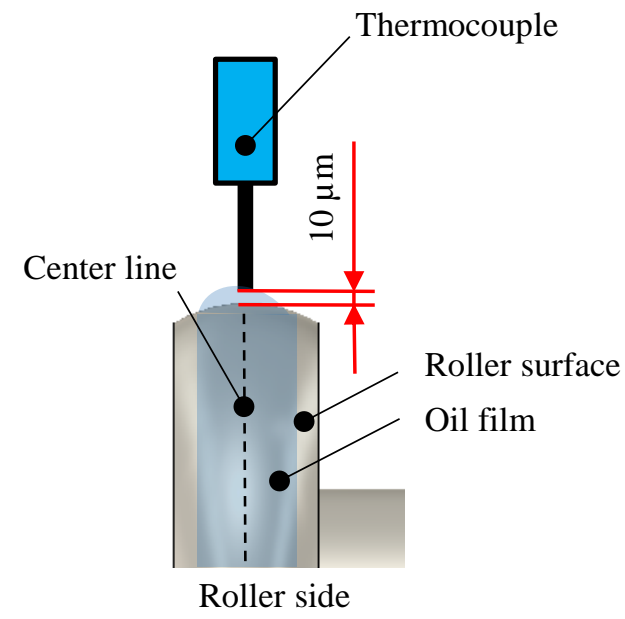

(b) Method of setting a thermocouple

Fig. 3 An oil temperature is measured with the thermocouple.

Table1 Experimental conditions of oil film temperature measurement

\begin{tabular}{l|l}
\hline \hline Rotational direction & Upward and Downward \\
\hline Peripheral velocity of the roller & $10.0 \mathrm{~m} / \mathrm{s}$ \\
\hline Peripheral velocity of the disk & $5.0 \mathrm{~m} / \mathrm{s}$ \\
\hline Creep ratio & $50 \%$ \\
\hline Pressing load & $1000 \mathrm{~N}$ \\
\hline Lubrication oil & $\mathrm{KTF}-1$ \\
\hline Thermal conductivity of oil & $0.2 \mathrm{~W} / \mathrm{m} \cdot \mathrm{K}$ \\
\hline Amount of supply oil & $1.75 \mathrm{~mL} / \mathrm{min}$ \\
\hline Supply oil temperature & $26.9^{\circ} \mathrm{C}($ Average) \\
\hline Ambient temperature & $27.3^{\circ} \mathrm{C}($ Average) \\
\hline
\end{tabular}

\subsection{Visualization of oil splashing}

Figure 4 shows the visualization method of oil splashing. In this experiment, the oil droplet splashing from the roller surface is visualized with the high-speed camera in front of the roller as shown Fig. 4(a). The area of the oil droplet was measured from the visualized image, and the ratio of oil splashing around the entire circumference of the roller was calculated. The ratio of oil splashing is defined are as follows:

$$
\beta=\frac{A_{1}}{A_{2}}
$$

where $\beta$ is the ratio of oil splashing, $A_{1}$ is oil droplet areas, and $A_{2}$ is a section area. Here, the method of measuring oil droplet area is described. The visualized image of oil splashing is binarized to clear oil droplet contour. On the binarized image, the roller is divided into 12 sections 30 degrees as shown Fig. 4(b). In a section, oil droplets area is measured by using image processing software (Image-J, National Institutes of Health). In this study, ratio of oil droplets area in a section was compared with other sections. Experimental conditions are the same as the experiment of the measurement of oil film temperature as shown in Table 1. 


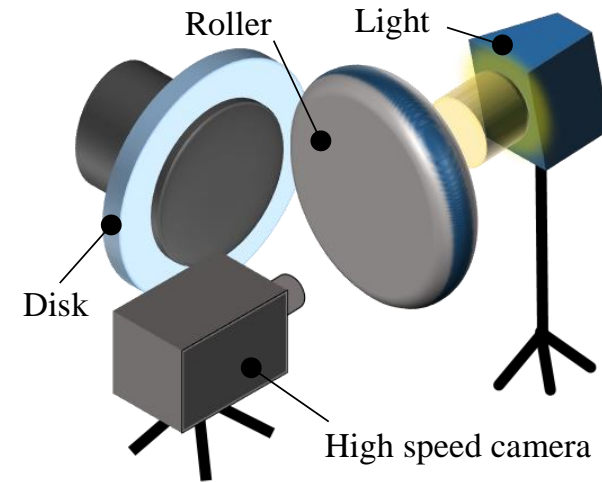

(a) Visualization of oil splashing with high speed camera

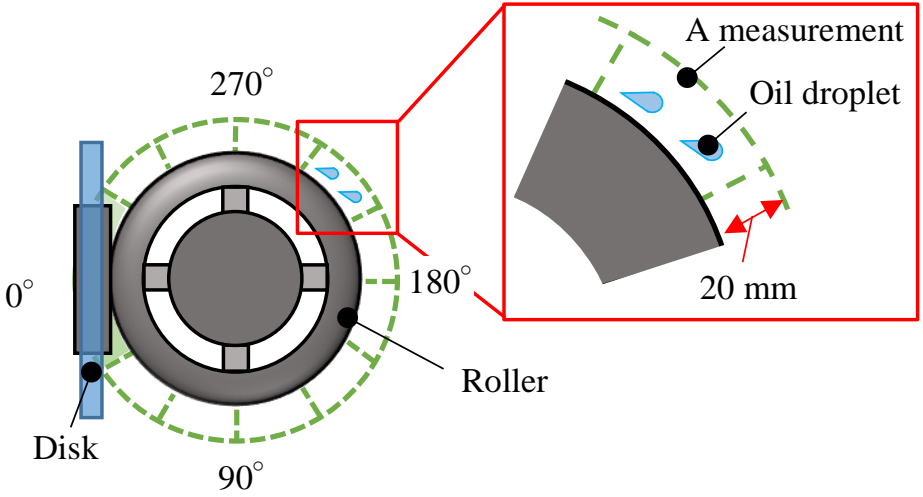

(b) Measurement section area of the oil droplet

Fig. 4 Visualization set-up and method of oil splashing

Table2 Experimental conditions of the visualization of the oil film distribution

\begin{tabular}{c|c}
\hline \hline Rotational direction & Upward \\
\hline Peripheral velocity of the roller & $2.0 \mathrm{~m} / \mathrm{s}$ \\
\hline Peripheral velocity of the disk & $2.0 \mathrm{~m} / \mathrm{s}$ \\
\hline Creep ratio & $0 \%$ \\
\hline Pressing load & $1000 \mathrm{~N}$ \\
\hline Lubrication oil & $\mathrm{KTF}-1$ \\
\hline Amount of the supply oil & $1.75 \mathrm{~mL} / \mathrm{min}$ \\
\hline
\end{tabular}

\subsection{Visualization of oil film distribution}

In this study, oil film distributions are measured by a new visualization method of Photochromism (Kuratuzi and Azetsu et al., 2018). Figure 5 shows the oil color change phenomenon by photochromism. The color of oil containing photochromic dye is changed by irradiating with ultraviolet light. This reaction simultaneously with the irradiation of ultraviolet light. A color density of thick oil film is higher than thin oil film as shown in Fig. 6 . The relationship between the oil film thickness and color density are as follows:

$$
\begin{aligned}
& \text { As }=\log _{10} \frac{I_{\text {after }}}{I_{\text {before }}} \\
& h=\alpha \cdot A s
\end{aligned}
$$

where $A s$ is the absorbance (the ratio of color density), $I_{\text {after }}$ is the light intensity of changed oil color, $I_{\text {before }}$ is the light intensity of nu changed oil color, $h$ is the oil film thickness, and $\alpha$ is the absorbance coefficient.

Thus, it is possible to visualize the oil film thickness distribution by using photochromism. The oil film shape on the roller surface is elucidated by the oil film thickness distribution. In addition, based on the results of the experiment, this paper considered the relationship between oil film shape on the roller surface and cooling.

Table 2 shows the experimental conditions of the visualization of the oil film distribution. In this experiment, an N2raser generator irradiated ultraviolet light rays to the oil film on the roller surface. Simultaneously, the irradiated portion is photographed with a high-speed camera. In addition, the color density is affected by output of ultraviolet light. However, the oil film thickness distribution is not affected because this N2 laser can irradiate the irradiated area with a uniform output. The process for taking a photograph of the top of the roller surface and side right of the roller surface is as shown in Fig. 7. 


\subsection{Visualization of the oil film around the contact point}

Figure 8 shows the experimental set up for the oil film visualization around the contact point. In the layout of the experimental apparatus, there is a mirror behind a disk and a high-speed camera above a mirror. The oil film around the contact point is reflected in the mirror and can be visualized with a high-speed camera. The experimental conditions are same as the visualization experiment of the oil film distribution as shown in Table 2.

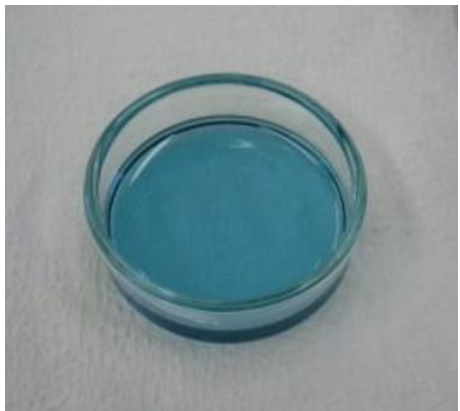

(a) Before

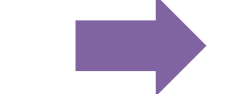

Ultraviolet light

Fig. 5 Photochromism is the phenomenon that the oil color is changed by irradiation the ultraviolet light ray. The oil (KTF-1) is changed the blue color to the purple.

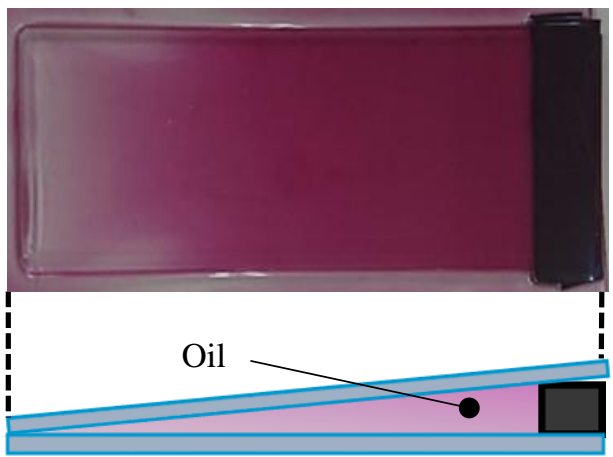

(a) Color picture of side view

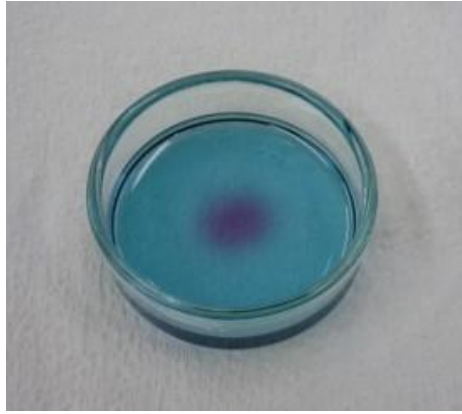

(b) After

Fig. 6 Relationship oil film thickness distribution and color density

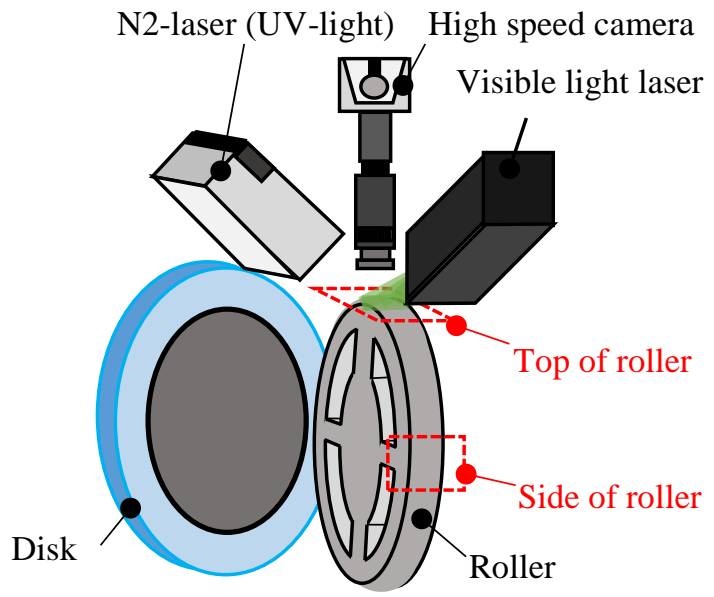

Fig. 7 Experimental set-up for visualization of the oil film distribution at the top and side of the roller

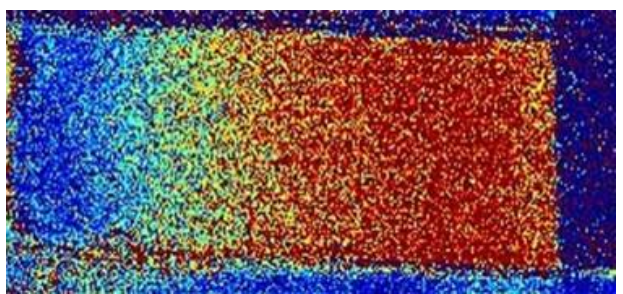

Oil film thickness

Thin Thick

(b) After processing the image 


\section{Results and discussion}

\subsection{Oil splashing and oil film temperature}

Figure 9 shows the experimental results of oil film temperature. From the results of the oil film temperature, in case of upward, the temperatures are lower than that of downward in a wide range of roller surface as shown in Fig. 9. It means that upward case is better than downward case for cooling. In addition, from the result of downward case, it is confirmed that the oil film temperature decreases rapidly after passing the contact point which is heat source. Therefore, around the contact point, the oil is cooled down by low temperature of supply oil. On the other hand, in the case of upward, the oil film temperature decreases gradually. Therefore, it is concluded that, in the case of downward, the supplied oil cannot cool the roller effectively around the contact point.

Figure 10 shows the experimental results of oil splashing ratio and Fig. 11 shows the visualization photo around the point of large amount of splash in each case. From Fig. 10 (a) and Fig. 11 (a), it is confirmed that the large amount of oil is splashed out immediately after passing the contact point caused by momentum and gravity. In addition, less oils are splashed at the other points. It means that almost all of amount of oil is splashed after passing the contact point. Therefore, it is considered that supplied oil is splashed out before absorbing large amount of heat. Moreover, in $60^{\circ}$ to $360^{\circ}$, the heat dissipation is mainly conducted against the ambient air because of less oil splash as shown in Fig. 12 (a). It is considered that the amount of dissipation is not so high, therefore the temperature in the case of downward becomes higher.

On the other hand, in the case of upward, the oil is splashed out gradually after $270^{\circ}$. Therefore, it is considered that the supplied oil is splashed out after absorbing roller heat. Because, the supplied oil adhere the heated oil film on the roller surface and then the heat is transmitted to the upper oil film gradually as shown in Fig. 12 (b). In addition, after splashing out at the top of roller caused by centrifugal force, next oil is gradually supplied from the side by the centrifugal force and the gravity as shown in Fig. 13. The supplied oil from the side does not pass the traction contact point, therefore the temperature is not so high. Due to this mechanism cycle, the roller can be cooled down effectively.

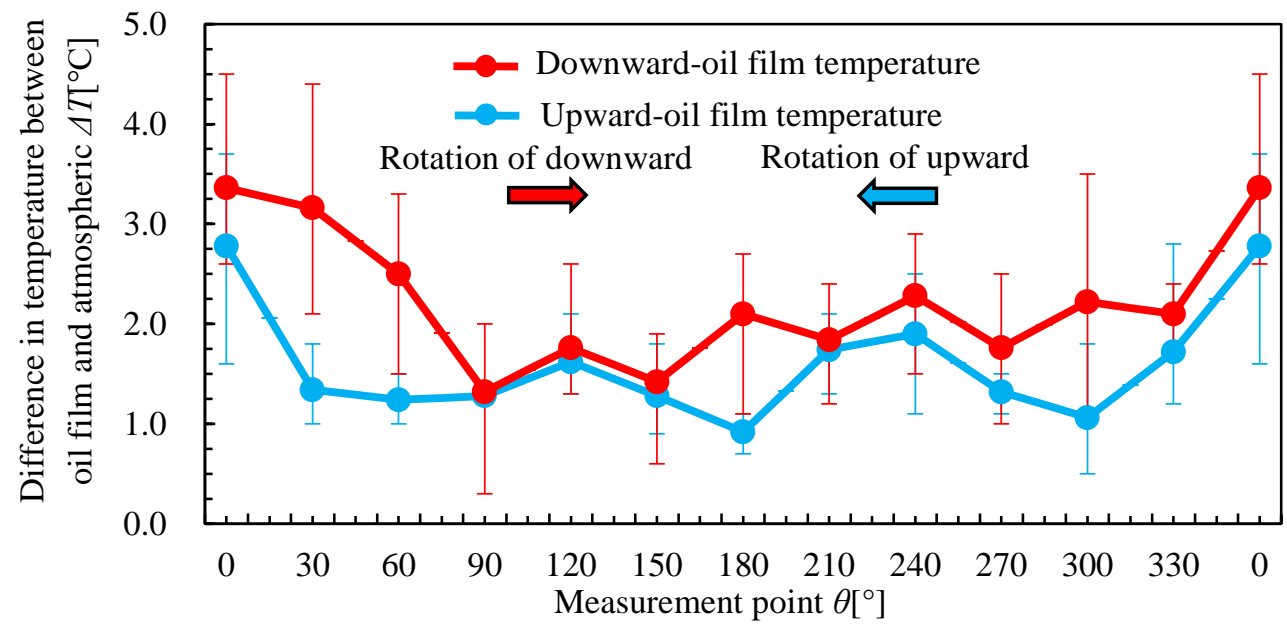

Fig. 9 Oil film temperature. The red means the downward and the blue means the upward

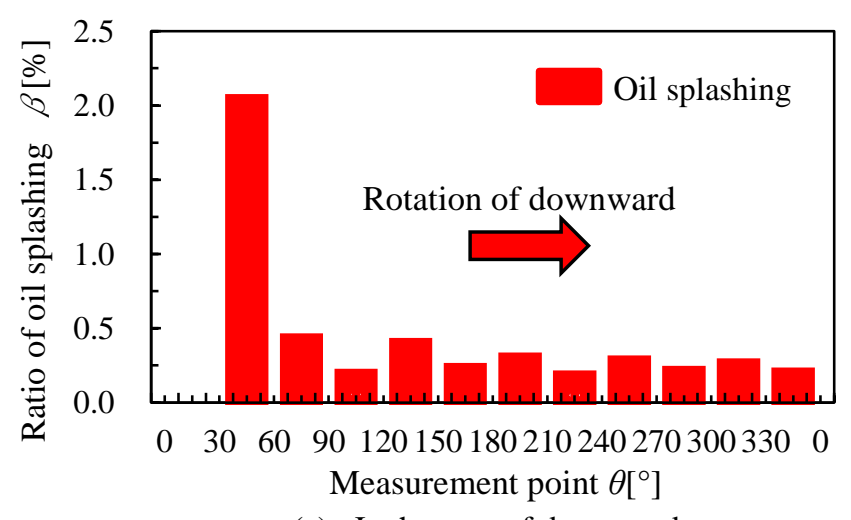

(a) In the case of downward

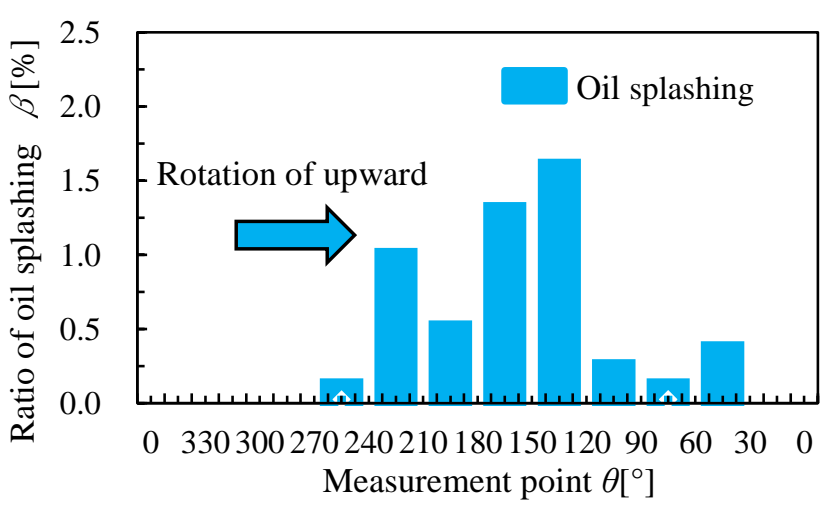

(b) In the case of upward

Fig. 10 Ratio of oil splashing. Fig. 10 (a) is the downward and Fig. 10 (b) is the upward. 


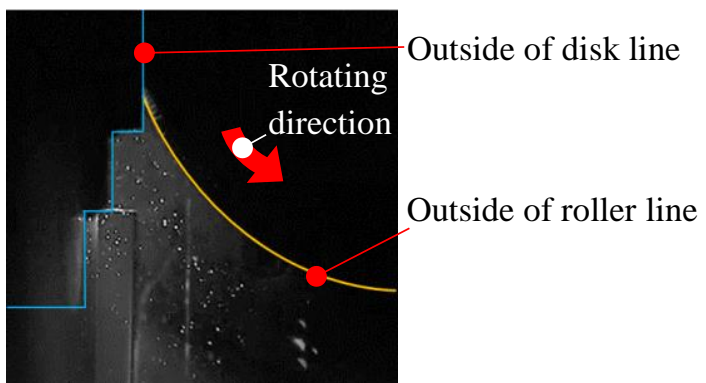

(a) In the case of downward

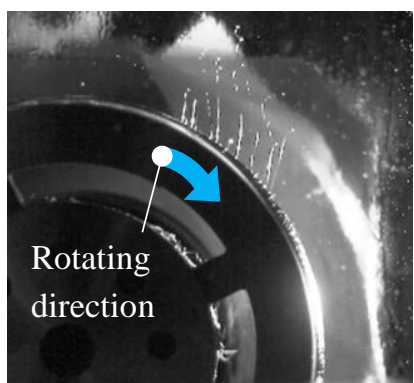

(b) In the case of upward

Fig. 11 The photo of the oil splashing. Fig. 11 (a) is the case of downward under the contact point.

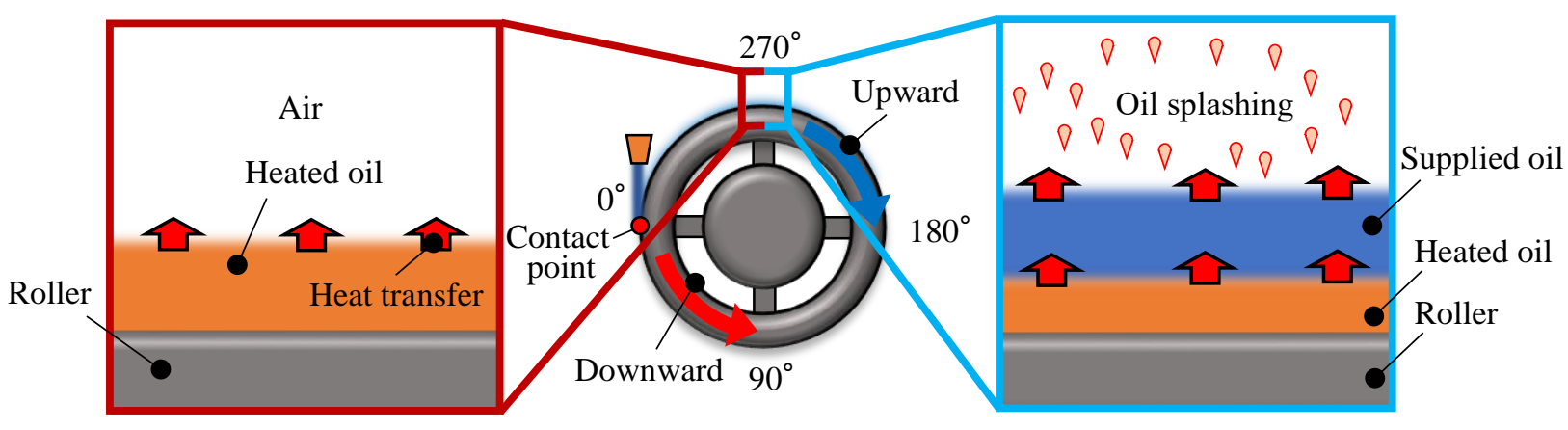

(a) In the case of downward

(b) In the case of upward

Fig. 12 The cooling mechanism. Fig. 12 (a) is the downward and Fig. 12 (b) is the upward.

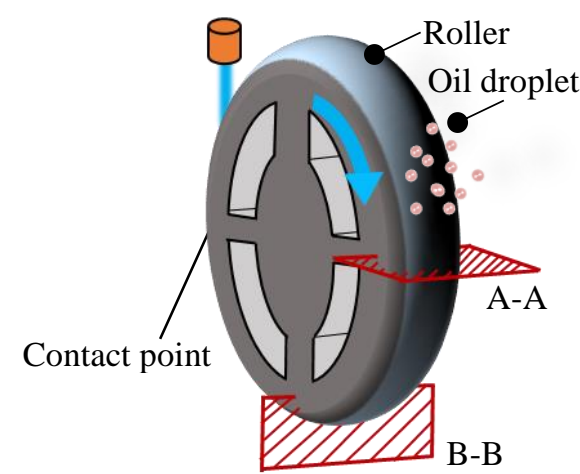

(a) Oil splashing from the roller

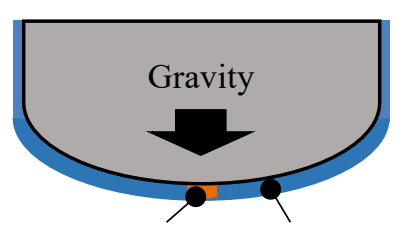

Heated oil Not heated oil

(b) Cutting model (A-A)

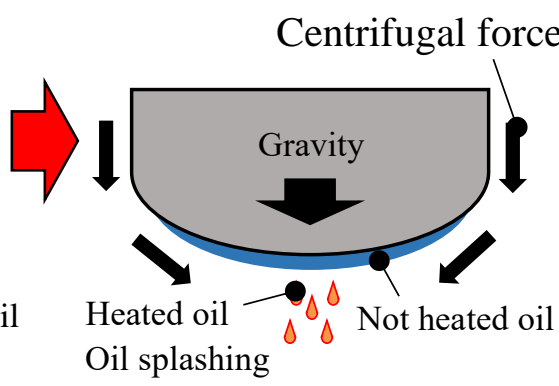

(c) Cutting model (B-B)

Fig. 13 Immediately after the oil passes through the contact point. Fig. 13 (b) is cutting model (A-A) and Fig. 13 (c) is cutting model (B-B) in Fig. 13(a).

\subsection{Oil film distribution}

In order to clarify the cooling mechanism, oil film distribution on the roller surface are measured. In this study, we used the new visualization method of photochromizm as mentioned in section 2.4.

Figure 14 shows the oil film distribution on the roller surface, and Fig. 15 shows the conceptual view of oil film shape on the roller surface. In Fig. 14, the red color means thick oil film and the blue color means thin oil film. From this result, it is found that the oil film thickness around the center line is thin compared with the side oil film thickness as shown in Fig. 14, and it is considered that the oil film is divided in half on the roller surface as shown Fig. 15. The reason is that the oil film is divided at the contact point as shown in Fig. 16. Consequently, in the case of upward, the oil is supplied to the contact point directly. However, the oil film on the center line is thin after passing the contact point and it is estimated that the cooling effect of heat absorption by the oil film is less. Thus, it is considered that the oil cooling effect is hindered by the oil film divided in half on the roller surface. Therefore, it is considered that the cooling 
effect will increase by increasing the amount of adhering oil around to the center on the roller surface.

A newly proposed roller geometry in this paper is shown in Fig. 17. Based on the above results and discussions, the new roller geometry was designed. In this geometry, there are twin grooves beside the center line, and it is considered that a large amount of oil would adhere around the center on the roller surface by the groves so that increased cooling effect of the oil film would be increased. A next step would be to test it and clarify the effectiveness of the grooves on cooling.

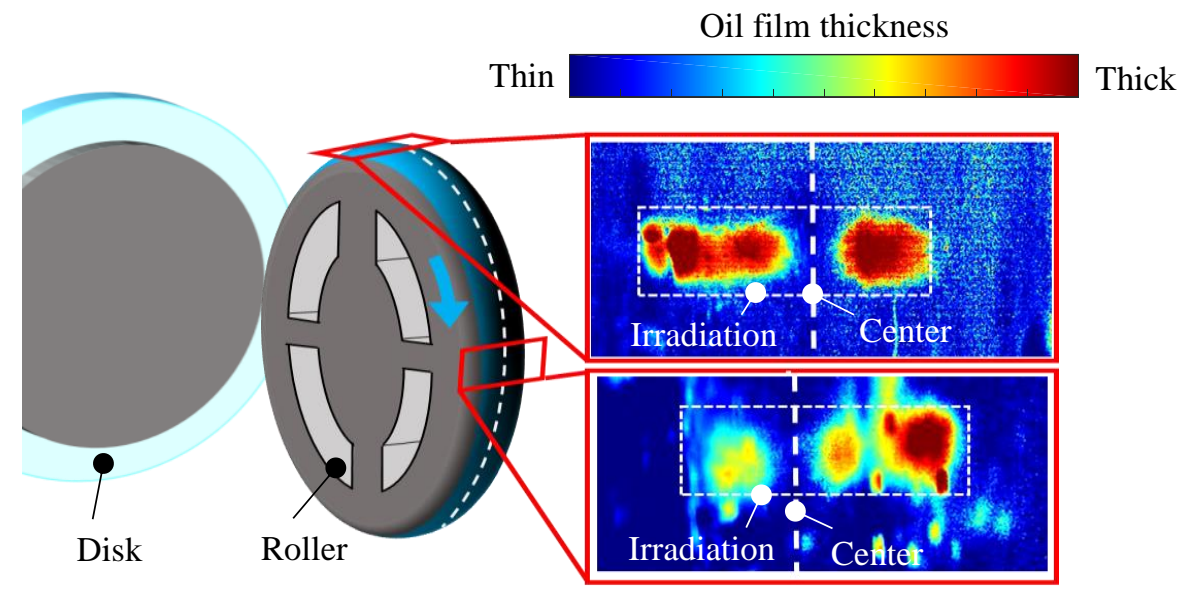

Fig. 14 The image shows the oil film distribution on the roller surface. The red color means thick oil film and the blue color means thin oil film.

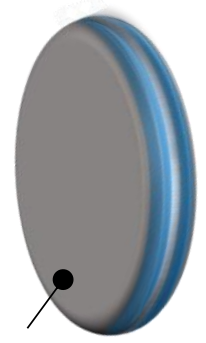

Roller

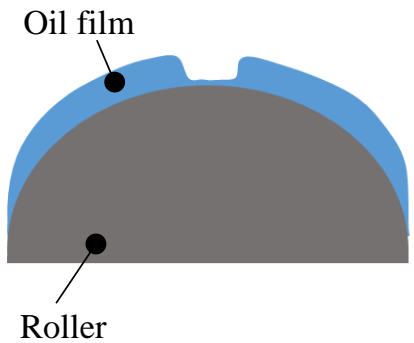

(b) Cutting view

(a) Overall oil film on the roller

(b) Cutting view

Fig. 15 Conceptual drawing of oil film shape on the roller surface. Fig. 15(a) shows overall drawing and Fig. 15(b) shows cutting view.

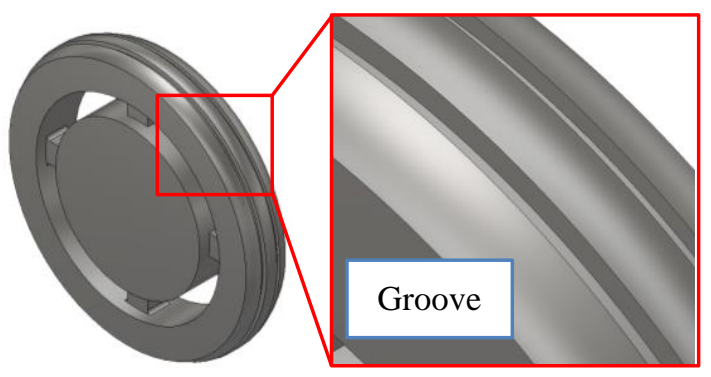

(a) New roller geometry

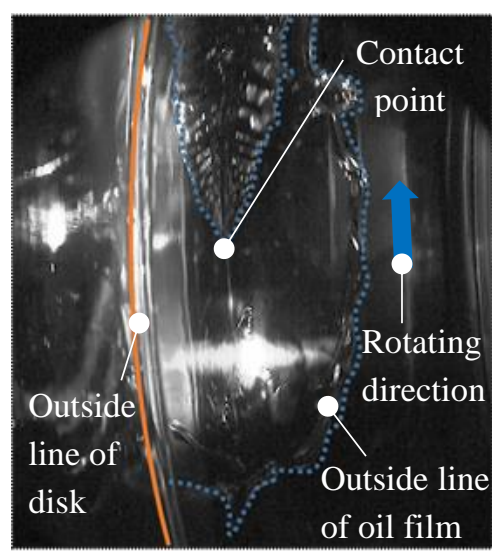

Fig. 16 Oil film around the contact point

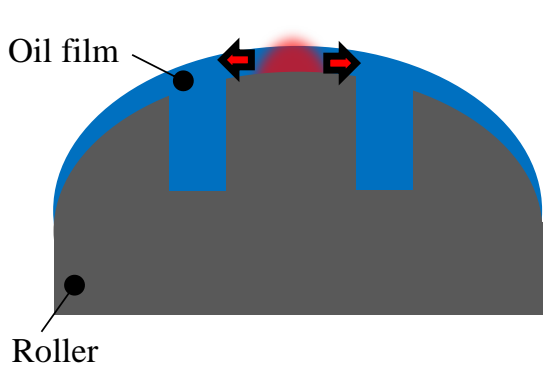

(b) Cutting view

Fig. 17 New roller geometry. Fig. 17(a) shows the whole of roller and Fig. 17(b) shows cooling effect of grooves. 


\section{Conclusion}

In this paper, the cooling effect of the oil film on the roller surface is examined on traction drives. The main conclusions are summarized as follows.

(1) In the experiment of an oil film temperature measurement, the oil film temperature in the case of upward is lower than that of downward. In the experiment of the oil splash-out visualization, it is confirmed that there is not the oil splashing around the top of roller in the case of upward and the supplied oil adheres on the roller surface. According to these results, it is considered that the heat of oil is absorbed by the supplied oil and the heated oil splashes.

(2) In this study, visualization of the oil film thickness distribution on the roller surface were realized by using photochromizm. As a result, it found that the oil film divides in half on the roller surface. Moreover, it is considered that cooling effect is less so that amount of oil is less at the center on the roller surface.

(3) According to these experimental results, it is considered that the cooling effect improves if the amount of oil adhered around the center line on the roller surface increases. Therefore, the authors considered that it would be improved if there are grooves on the roller surface. In the future, we will try to examine through the experiment and the thermal CFD analysis.

\section{References}

A. Dewinter, H. Blok, Fling-Off Cooling of Gear Teeth, Transactions of the ASME Journal of Engineering for Industry, Feburuay1974, pp.60-70

Kuratuzi, K., Kitajima, I., Azetsu, A., Tamaki, S., Nangou, T., Measurement of Oil Behavior around Piston Photochromic Visualization Technique, International Journal of Automotive Engineering (IJAE) Vol. 50, No.1 (2019), pp.55-60

Mchida, H., Characteristics of Traction Drive Power Transmissions, Journal of the Japan Society for Precision Engineering, vol. 56, No.9 (1990), pp.1597-1601

Obata, F., Ymaura, H., Tanaka, H., Morioka, K., Mitsudo, H., Gentrifugal Lubrication of Spur Gears (1st Report, Behavior and Flow Rate of Oil Jet), Transactions of the Japan Society of Mechanical Engineers, Series C vol. 60, No.573 (1994), pp.297-304

Suwa, T., Ochiai, M., Hashimoto, H., Study on Supply Direction of Lubrication Oil by Using CFD Analysis in Traction Drive, Proceedings of ICMDT2019

Tanaka, H., Power Transmission of a Cone Roller Toroidal Traction Drive (1st Report, Speed and Torque Transmission Efficiencies), Transactions of the Japan Society of Mechanical Engineers, Series C vol. 53, No.491 (1987), pp.15001506

Yamada, T., Mitui, J., Fujiwara, K., Okui., T., Cooling the Gear by the Lubrication oil, Transactions of the Japan Society of Mechanical Engineers, Series C vol. 47, No.419 (1981), pp.901-908 